\title{
On the breaking of long, axisymmetric liquid bridges between unequal supporting disks at minimum volume stability limit
}

\author{
José Meseguer, José L. Espino, José M. Perales , Ana Laverón-Simavilla
}

IDR/UPM. E.T.S.I. Aeronánticos, Unversidad Politecnica de Madnd, 28040 Madrid. Spam

\begin{abstract}
The stability of axisymmetric liquid bridges held between non-equal circular supporting disks, and subjected to an axial acceleration, has been analyzed both theoretically and experimentally. Some characteristics of the breaking process which takes place when the stability limit of minimum volume is reached (mainly the dependence with the disks separation of the volume of the liquid drops resulting after the liquid bridge breakage) have been theoretically studied by using standard asymptotic expansion techniques. From the analysis of the nature of the unstable equilibrium shapes of minimum volume at the stability limit it is concluded that the relative volume of the main drops resulting from the liquid bridge rupture drastically change as the disks separation grows. Theoretical predictions have been experimentally checked by working with very small size liquid bridges (supporting disks being some 1 millimeter in diameter). the agreement between theoretical predictions and experimental results being remarkable.
\end{abstract}

Keywrords: Liquid bridge; Surface tension; Capillary instability

\section{Introduction}

A liquid bridge consists of an isothermal mass of liquid of volume $\tilde{V}$ held by surface tension forces between two parallel, coaxial circular disks that are separated by a distance $L$. The disk radii are $R^{+}$and $R^{-}$, respectively (see Fig. 1).

Liquid bridges are present in a large variety of physical and technological situations, and a great effort has been done in the last decades to determine equilibrium shapes and stability limits for a wide range of liquid bridge configurations. The simplest non-trivial (non-cylindrical) liquid bridge configuration consists of an axisymmetric drop of liquid spanning between two parallel, coaxial, equal in diameter solid disks, in absence of body forces. Early stability studies concerning basic configurations were published three decades ago (Haynes [1]. Erle. Gillette and Dyson [2], Gillette and Dyson [3]). although the most relevant result conceming the stability of such configuration were published at the end of the decade of the seventies (Martinez [4]), and in the last two decades (Sanz and Martinez [5]. Russo and Steen [6]. Boucher and Jones [7], Lowry and Steen [8], Slobozhanin, Alexander and Resuick [9], amongst other). Conceming the influence of axial gravity, different attempts have been made to calculate the minimum volume stability limits as well as the maximum volume stability limits both from a theoretical and an experimental point of view [10-12], being this problem extensively analyzed in the nineties by Slobozhanin and Perales [13]. The influence of non-equal coaxial disks on the stability limits has been investigated in the last twenty years. At the beginning the effort was concentrated on the stability limit of minimum volume (Martinez and Perales [14]), the stability limits of maximum volume being reported more recently by Slobozhanin, Gómez and Perales [15]. 


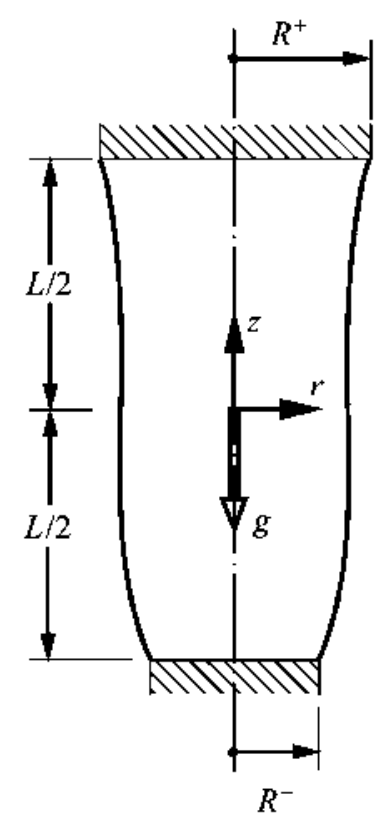

Fig. 1. The liquid bridge sct-up.

The combined effect of both unequal disks and axial gravity on liquid bridge stability was first studied by Mescguer [16] through a bifurcation analysis, and different improvements to such stability study, either theoretical or experimental, have been published later [17-20]. The most comprehensive results published up to now on the combined influence of unequal disks and axial gravity can be found in Perales, Meseguer and Martine $\%$ [21], where minimum volume stability limits are analyzed both theoretically and experimentally, and in Slobozhanin and $\Lambda$ lexander |22|, where the complete stability diagrams are calculated.

Other axisymmetric (solid body rotation) and non-axisymmetric cffects (latcral acecleration, non-coaxial disks) have been also taken into account to analyze liquid bridge stability (a review of the literature dealing with these effects can be found in Meseguer et al. [23]). An asymptotic study on the influence of non-circular disks on the stability limit of cylindrical volume liquid bridges has been published recently [24]. In addition, a fairly significant number of papers dealing with the application of either flow stabilization $|25,26|$, acoustic stabilization $[27,28 \mid$, electric field stabilization $|29-33|$ or magnetic field stabilization $[34,35]$ have been published.

Besides, therc have also becn numcrous investigations of liquid bridge dynamics. The nonlinear dynamics and breakage of liquid bridges was first studied some twenty years ago, using inviscid, one-dimensional models $[36,11,17]$. Sirignano and Mehring [37] have recently published a review of the theories commonly used in liquid jet breaking, and an historical review of the most representative publications concerning the breaking of liquid bridges can be found in Zhang, Padgett and Basaran |38]. That review has been completed with a very recently published paper on this topic [39].

The stability (and its effect on the breaking dynamies) of long axisymmetric liquid bridges held between unequal, parallel circular supporting disks subject to axial acceleration can be theoretically analyzed by using an analytical approximation based on the standard bifurcation theory (Lyapunov-Schmidt technique [40]). According to this method, the expression for both the stable and the unstable equilibrium interface shapes is $f(z)=1+\varepsilon \sin (z / \Lambda)$, where $\varepsilon$ is a small parameter (its value depending on the perturbations acting on the liquid bridge) which measures the magnitude of the interface deformation. Therefore, when the instability develops, the liquid bridge interface bulges in one half of the liquid column and neeks in the other (the instability is antisymmetric with respect to the mid-plane parallel to the disks).

According to this behavior, any perturbation leading to an antisymmetric deformation of the interface, as axial gravity or unequal coaxial disks, will force a neck in the liquid column which will determine the further breaking process. When two antisymmetric effects, like the ones stated above, act in opposite directions, the interface deformations associated to each one of the parameters will also be opposite, so that the interface neck will be in the upper or in lower half of the liquid column depending on the relative importance of the parameters involved. Although this problem was first analyzed, more than a decade ago, both numerically and experimentally [20,21], and the main features of the breaking process were outlined, no explanation on the different effects involved in the development of instabilities was given in these papers. Refined asymptotic analysis shows that for fixed values of the parameters under consideration (unequal disks and axial gravity), their relative importance depend 
on the disks separation. Therefore the (relative) volume of the drops resulting after the liquid bridge rupture must also depend on the disks separation.

In the following an asymptotic analysis of the unstable equilibrium shapes of liquid bridges at minimum volume stability limit is presented. Such study leads to a very simple expression for the stability limit which reveals that for the liquid bridge configuration under consideration. there is a strong dependence of the equilibrium shapes on the disk separation. The neck of the liquid column moves along the liquid bridge, from the upper to lower half and vice versa, as the disk separation varies. The neck migration is very accentuated when the fluid configuration is close to the local minimum volume which corresponds to the stability limit eurve [20-22]. This implies a drastic change in the breaking dynamies of the liquid column. The liquid bridge breaks in two main drops with unequal relative volumes. For a disk separation smaller than the critical (the local minimum volume) the drop anchored to the upper disk is larger. As the disk separation increases the neck moves upwards. and when the separation is larger than the critical, the larger drop is at the bottom disk.

Such behavior has been experimentally checked by working with very small size liquid bridges. In addition. asymptotic resul ts are compared with the exact theoretical ones [21,22]. and with experimental results already published [20.21].

\section{Analytical approach}

Equilibrium shapes of axisymmetric liquid bridges are deseribed by the Young-Laplace equation, which in dimensional variables takes the form:

$$
\sigma \tilde{M}(\tilde{F})+\widetilde{P}-\Delta \rho g \tilde{z}=0
$$

where $\tilde{M}(\tilde{F})$ is twice the mean curvature of the interface, $\tilde{F}=\tilde{F}(\tilde{z})$ is the equation for the axisymmetric liquid bridge interface, $\tilde{F}$ is a constant related to the origin of pressure, $\Delta \rho$ is the difference in density between the liquid bridge and the surrounding fluid, $\sigma$ is the interfacial tension between the bridge and the surrounding fluid and $g$ is the axial aceeleration. The boundary conditions and constraints express the constant value of the liquid bridge volume, and that the bridge surface is anchored or pinned to the edges of the supporting disks.

Taking $R_{0}=\left(R^{+}+R^{-}\right) / 2$ as the characteristic length of the problem. and defining $F=\tilde{F} / R_{0} \cdot z=\tilde{z} / R_{0}$. Eq. (1) can be recast in dimensionless form as $M(F)+P-B z=0$, where $M(F)=\left[F F_{z z}-1-\left(F_{z}\right)^{2}\right] \cdot\left[1+\left(F_{z}\right)^{2}\right]^{-3 / 2} \cdot F^{-1}$. The constant volume condition takes the form

$$
\int_{-A}^{A} F^{2} \mathrm{~d} z=2 A V
$$

and the boundary conditions at the disks are $F( \pm A)=1 \pm h$, where, $h=\left(R^{+}-R^{-}\right) /\left(R^{+}+R^{-}\right)$. In this formulation $P$ has been made dimensionless using $\sigma / R_{0}, B$ is the axial Bond number, $B=\Delta \rho g R_{0}^{2} / \sigma, A=L /\left(2 R_{0}\right)$ is the slenderness and $V=\widehat{V} /\left(\pi R_{0}^{2} L\right)$ is the dimensionless volume. The subseript $z$ indicates derivatives with respect to this variable.

Critical points result after linearisation of the problem formulation above stated. It is well known that for the case $B=h=0$. $V=1$, the problem under consideration has the trivial equilibrium solution $F=1, P=1$ for any $A$. Then, the introduction of the expansions $F(z)=1+\varepsilon \hat{f}(z)+\mathrm{O}\left(\varepsilon^{2}\right), P=1+\varepsilon \hat{p}+\mathrm{O}\left(\varepsilon^{2}\right)$, where $\varepsilon$ stands for the magnitude of the deformation of the interface. allows us to calculate $\hat{f}(z)$ and thus other equilibrium solutions after neglecting $\mathrm{O}\left(\varepsilon^{2}\right)$ terms. The process used to obtain the variation of the maximum stable slenderness due to the different effects under consideration has been described elsewhere [41], so that in the following paragraphs only the main features of such procedure are outlined (specific details on the problem here analyzed can be obtained upon request to the authors).

Non-trivial (different from zero) solutions of the linear problem only exist for discrete values of the liquid bridge slenderness. $\Lambda=\pi$ being the smallest value of $A$ for which a non-trivial solution exists (at $A=\pi$ the transition from stable to unstable equilibrium shapes occurs). Within this approximation, the departure from the cylinder of the unstable equilibrium shapes is given by $\hat{f}(z)=\sin (\pi z / \Lambda), \hat{p}=0$, which is the solution that must be perturbed to calculate the variation of the maximum stable slenderness. In the asymptotic approximation, a new variable which nomalizes boundary conditions, $x=\pi z / \Lambda$, is introduced along with the parameter $\lambda=1-\Lambda / \pi$, and higher order terms than those considered in the linear problem are retained. Let $f(x)$ and $p$ represent these higher order terms of the interface shape and the pressure. respectively. The new expansions for $F$ and $P$ are then $F(z)=1+\varepsilon \sin x+f(x), P=1+p$. which. after substitution in the problem formulation yields the new problem

$$
M(1+\varepsilon \sin x+f(x))+1+p-B x(1-\lambda)=0
$$

with 


$$
\begin{aligned}
& f( \pm \pi)= \pm h, \\
& \int_{-\pi}^{\pi}[1+\varepsilon \sin x+f(x)]^{2} \mathrm{~d} x=2 \pi(1+v),
\end{aligned}
$$

where $v=V-1$. Note that the curvature of the interface, $M(F)$, is now computed in terms of the variable $x$, so that $M(F)$ now includes $\lambda$ as a parameter. This formulation requires an additional condition to uniquely define the parameter $\varepsilon$; such condition is:

$$
\int_{-p}^{p} f(x) \sin x \mathrm{~d} x=0
$$

The problem defined by Eqs. (2)-(5) allows us to calculate $f(x)$ and $p$ in terms of the small parameters $\varepsilon, \lambda . v, h$ and $B$ by using the bifurcation equation (the Lyapunov-Schmidt technique). In this technique. instead of Eq. (2) the equation to be solved is

$$
M(1+\varepsilon \sin x+f(x))+1+p-B x(1-\lambda)+\phi \sin x=0,
$$

and from the Implicit Function Theorem [40] it can be demonstrated that, at least in a neighborhood of $\varepsilon=\lambda=v=h=B=0$, the expression (6) with conditions (3)-(5) uniquely define $f(x), p$ and $\phi$. which can be expressed as series expansions of the involved small parameters, i.e::

$$
\phi(\varepsilon, \lambda, v, h, B)=\sum_{i=1}^{5} \varepsilon_{i} \phi_{i}+\sum_{i=1}^{5} \sum_{j=1}^{5} \varepsilon_{i} \varepsilon_{j} \phi_{i j}+\sum_{i=1}^{5} \sum_{j=1}^{5} \sum_{k=1}^{5} \varepsilon_{i} \varepsilon_{j} \varepsilon_{k} \phi_{i j k}+\cdots
$$

Similar expressions can be written for $f(x)$ and $p$. In these expansions the terms $\varepsilon_{i}$ represent the problem goveming parameters (i.e., $\varepsilon_{1}=\varepsilon, \varepsilon_{2}=\lambda, \varepsilon_{3}=v, \varepsilon_{4}=h, \varepsilon_{5}=B$ ), and the different unknowns are symmetric with respect to their indices (i.e., $\phi_{i j}=\phi_{j i}$ ). The introduction of the asymptotic expansions for $\phi, p$ and $f(x)$ into Eq. (6) yields different sets of problems of order $\varepsilon$, order $\varepsilon^{2}$, order $\varepsilon^{3} \ldots$ which allows the calculation of the different functions and coefficients involved $\left(f_{i}(x), f_{i j}(x), f_{i j k}(x), \ldots, p_{i}, p_{i j}, p_{i j k}, \ldots, \phi_{i}, \phi_{i j}, \phi_{i j k}, \ldots\right)$. Obviously, such solutions will correspond to the solution of the original problem formulation if and only if the parameters involved satisfy the bifurcation equation $\phi(\varepsilon, \lambda, v, h, B)=0$. Hence, once the relevant problems are solved, the bifurcation equation becomes

$$
-\frac{h}{\pi}+B+\varepsilon \lambda+\frac{1}{2} \varepsilon v-\frac{3}{4} \varepsilon^{3}-\lambda\left(B+2 \frac{h}{\pi}\right)+\frac{1}{16} \varepsilon^{2}\left(B+15 \frac{h}{\pi}\right)-\frac{69}{64} \varepsilon^{5} \cdots=0,
$$

where only the non-zero leading terms for each parameter, plus non-zero terms of the following order (those in $\lambda B, \lambda h, \varepsilon^{2} B$. $\varepsilon^{2} h$ and $\varepsilon^{5}$ ) have been written. Note that, according to expression (8), $v$ and $\lambda$ are of order $\varepsilon^{2}$, whereas $B$ and $h$ are of order $\varepsilon^{3}$. Therefore, all the underlined terms are of order $\varepsilon^{5}$. Thence, to this approximation the reduced slenderness is

$$
\lambda=\frac{1}{B+2 h / \pi}-\varepsilon\left[B-\frac{h}{\pi}+\frac{1}{2} \varepsilon v-\frac{3}{4} \varepsilon^{3}+\frac{1}{16} \varepsilon^{2}\left(B+15 \frac{h}{\pi}\right)-\frac{69}{64} \varepsilon^{5}\right] .
$$

The stability limit is defined by $\lambda=\lambda_{\min }$, or $\Lambda_{\max }=\pi\left(1-\lambda_{\min }\right)$. Thus, taking $\mathrm{d} \lambda / \mathrm{d} \varepsilon=0$, if higher order terms (underlined terms) are omitted. we find that to leading order $\Lambda_{\max }$ is given by the well known expression [16,41]:

$$
\Lambda_{\max }=p\left\{1-\left(\frac{3}{2}\right)^{4 / 3}\left[B-\frac{h}{\pi}\right]^{2 / 3}+\frac{1}{2} v\right\} .
$$

Expression (10) shows the quantitative and the qualitative influence of the different parameters, which is determined by the exponent of each group of terms in this equation (10). Such influence can be understood considering the effect of the imposed perturbation on the necking of the liquid column. In effect, the expression for both stable and unstable equilibrium interface shapes is of the form $f(x)=1+\varepsilon \sin x$. Therefore, when the instability develops. the liquid bridge interface bulges in one half of the liquid column and necks in the other (the instability is antisymmetric with respect to the mid-plane parallel to the disks). According to this behavior, any perturbation leading to an antisymmetric deformation of the interface will decrease the maximum stable slenderness, the reduction being proportional to the two-thirds power of the perturbation. Note that since the difference in disk radii causes an antisymmetric deformation in the same manner as the Bond number, these two effects can be additive or subtractive, and can even cancel one another (the relative influence of these parameters on the drops resulting after liquid bridge breakage has been analyzed elsewhere [11.17.20]). 
Perturbations that cause a symmetric deformation (with respect to the mid-plane) of the interface, such as those duc to a reduction in volume, may also reduce the maximum stable slenderness. However, these kind of perturbation are less critical being the reduction in $A_{\text {max }}$ linear in the volume, $v$.

Expression (10) is only valid close to the reference liquid bridge configuration (defined by the Rayleigh stability limit, $A=\pi, V=1, B=h=0$ ), and far from this configuration equation (10) gives only a rough approximation of the influence on the stability limit of the different parameters considered. For instance, if $B$ and $h$ arc fixcd, Eq. (10) predicts a linear dependenec of the maximum stable slenderness on the liquid bridge volume, and no additional information can be obtained from this approximation on the influence of the liquid bridge volume on the breaking process that will occur once the stability limit is reached. In effech, if the underlined terms in $\mathrm{kq} .(9)$ are omilled, the reduced slenderness becomes

$$
\lambda=-\frac{1}{\varepsilon}\left(B-\frac{1}{\pi} h\right)-\frac{1}{2} v+\frac{3}{4} \varepsilon^{2},
$$

therefore $\mathrm{d} \lambda / \mathrm{d} \varepsilon=0$ implies $\varepsilon=0$ if $B-h / \pi=0$, or $\varepsilon=-|2(B-h / \pi) / 3|^{1 / 3}$ in the case $B-h / \pi \neq 0$. This last solution means that at the stability limit, irrespective of the value of the reduced slenderness, the liquid bridge would bulge at its lower half part (close to the small disk) when $B>h / \pi$, so that in this case a large drop would be formed at the lower (smaller) disk, and a small drop at the upper (larger) one after the liquid bridge rupture. On the other hand, when $B<h / \pi$, Eq. (11) predicts that at the stability limit the liquid bridge would neck at its lower half part, so that the size of the drops resulting after breaking would be just in opposition to the ones resulting when $B>h / \pi$. Obviously, these conclussions are far from the real behavior. Numerical and experimental results show that, for the liquid bridge configurations under consideration, the drops arrangement resulting from the liquid column breakage strongly depends on the slenderness $[11,17,20 \mid$.

To analyze the influence of the volume on both the stability limit and the breaking process other terms than the leading terms must be retained in the bifurcation equation (expression (9)). In this case, the calculation of the roots of the equation $\mathrm{d} \lambda / \mathrm{d} \varepsilon=0$ requires a much more involved algebra. The dependence of the parameter $\varepsilon$ on the reduced slenderness $\lambda$, as resulting from cxpression (9), is shown in Fig. 2. These results correspond to the cases $B=0.05, h=0.1$ (so that $B-h / \pi>0$ ), the reduced volume bcing $v=-0.4$ (Fig. 2(a)) or $v=-0.1$ (Fig. 2(b)). As it is well known, for cach liquid bridge configuration the stability limit is defined by the value $\lambda_{\min }$ where $\mathrm{d} \lambda / \mathrm{d} \varepsilon=0$. $\Lambda$ s shown in Fig. 2 the bifurcation equation has three roots for $\lambda>\lambda_{\text {min }}$
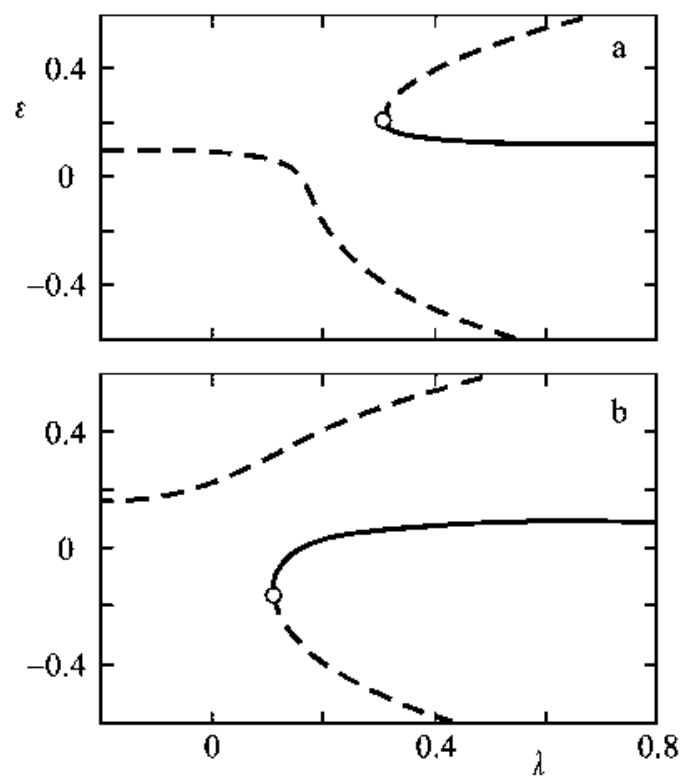

Fig. 2. Sketch of the equilibrium solution (departure from the cylinder $s$ ) as a function of the reduced slenderness $\lambda$, for two values of the reduced volume: (a) $v=-0.4$, and (b) $v=-0.1$. Thesc results have been obtained by using expression (9) and correspond to liquid bridges between unequal disks $(h=0.1)$ subject to an axial Bond number $B=0.05$. The stable solution is ploted in solid line and the unstable solutions in dashed line. In each sketch the circle indicates the stability limit, $\lambda_{\min }$, where $\mathrm{d} \lambda / \mathrm{d} \varepsilon=0$.

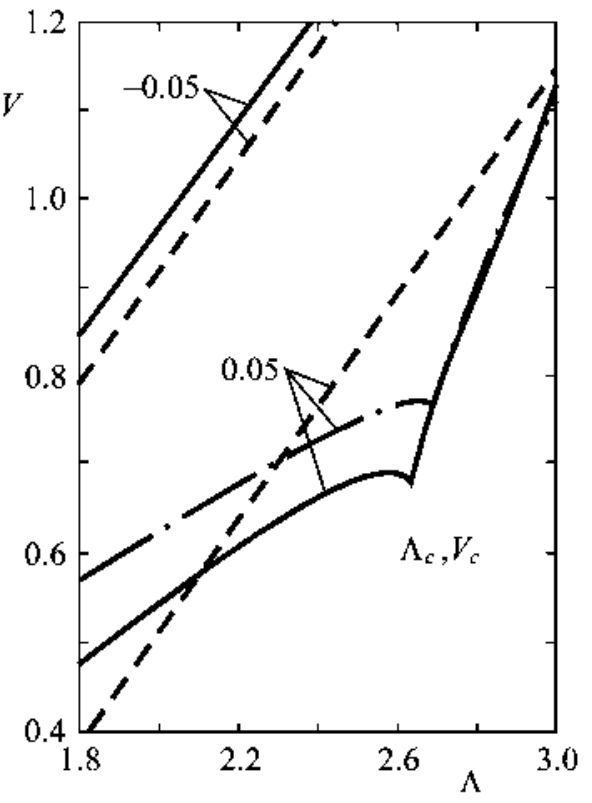

Fig. 3. Variation with the slenderness $A$ of the minimum volume $V$ of liquid bridges between unequal disks, $h=0.1$, subjecl to an axial Bond number. Dashed lines correspond to the results provided by expression (11) whereas solid curves represent results obtained by using expression (9). Dot-dashed line indicate results obtained by using the exact theoretical models $[21,22]$. Numbers on the curves indicate the value of the Bond number. 


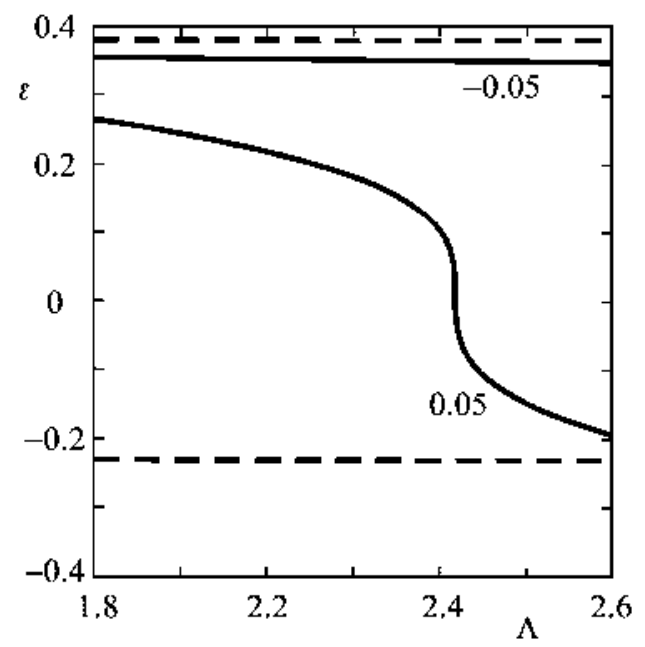

Fig. 4. Variation with the slenderness $A$ of the parameter 8 that measures the magnitude of the interface deformation or the unstable equilibrium shapes of liquid bridges between unequal disks, $h=0.1$, subject to axial Bond number. Dashed lines correspond to the results provided by expression (11) whereas solid lines represent results obtained by using expression (9). Numbers on the curves indicate the value of the Bond number.

(that means $\Lambda<\Lambda_{\max }$ ). The root represented by the solid lines in Fig. 2 is the root with the smallest $|\varepsilon|$ for $\lambda>\lambda \min$, and corresponds to the stable solution, whereas the other two real roots appearing for $\lambda>\lambda_{\text {min }}$ represent unstable solutions (for $\lambda<\lambda_{\min }$ there is only one real root which represents an unstable configuration).

Stability limits resulting from this asymptotic analysis are shown in Fig. 3. Five curves are represented in this plot: the straight (dashed) lines correspond to the results provided by expression (11) whereas solid lines represent results obtained by using expression (9). Dot-dashed line corresponds to exact theoretical results already published [21,22]. In all cases the valuc of the parameter that measures the disks incquality is $h=0.1$, the values of the Bond number being those labeled in the curves. Results obtained with Eq. (9) are much more similar to the ones resulting either from numerical or experimental analyses [20-22]. It must be pointed out, as it is clearly shown in Fig. 3, that for fixed $h$ and $B$ (provided $B>0$ ), the quantity $V$ at the stability limit boundary allains a local minimum al $V=V_{c}$ for a value $A=A_{C}$.

The variation of the parameter $\varepsilon$ with the slenderness $\Lambda$ that satisfies the equation $\mathrm{d} \lambda / \mathrm{d} \varepsilon=0$ is shown in Fig. 4 for the same liquid bridge configurations considered in Fig. 3. The line type indicates the expression used to solve $\mathrm{d} \lambda / \mathrm{d} \varepsilon=0$ : the results obtained using equation (11) are plotted with dashed lines, and the results obtained using Eq. (9) are represented using solid lines. The numbers on the eurves identify the values of the Bond number $B$. As it can be observed, as already anticipated, when Eq. (11) is used the value of the root remains constant, irrespective of the value of the liquid bridge slenderness, in other words, it does not depend on the liquid bridge volume. The behavior is rather different, and much more similar to the results provided by experimental evidence, when expression (9) is used instead of $\mathrm{Eq} .(11)$.

In this case the root of $\mathrm{d} \lambda / \mathrm{d} \varepsilon=0$ is always positive when $B-h / \pi<0$, because when this inequality holds both effects, Bond number and disks inequality are in phase, so that both perturbations force the necking of the liquid column in the same way (the neck appears close the bottom, smaller disk). Thus, when the breaking of the liquid column takes place, the resulting configuration is a large drop at the top (larger) disk, and a small one at the bottom (small) disk.

On the other hand, when $B-h / \pi>0$ the root of $\mathrm{d} \lambda / \mathrm{d} \varepsilon=0$ can be positive or negative, depending on the value of the slenderness. For $A<\Lambda_{c}\left(V<V_{c}\right)$ such root is positive (see Fig. 2(a)), whereas it becomes negative when $A>\Lambda_{c}\left(V>V_{c}\right)$. This result indicates that the position of the neck of the unstable equilibrium shapes varies as one moves along the minimum volume stability curve. When the slenderness is small $\left(A<A_{c}\right)$, after the liquid column rupture a large drop is formed at the top (larger) disk, and a small drop at the bottom disk. Since the Bond number effect (which is positive) tends to neck the upper half part of the liquid column, whereas disks incquality tends to neck the lower part, it can be concluded that for low values of the slenderness the effect of disks inequality dominates over the Bond number effect.

The contrary happens when $\Lambda>\Lambda_{c}$, the root of $\mathrm{d} \lambda / \mathrm{d} s=0$ is negative (see Fig. 2(b)), the unstable equilibrium shapes neck close the top disk, and, in consequence, after breaking there is a large drop at the bottom disk and a small drop at the top one. This implies that, when liquid bridge volumes are large, the dominant effect is the l3ond number. The dependence on the slenderness of the volume of the drops, with a sudden change at $A=\Lambda_{c}$, has been experimentally corroborated (see the following section). 


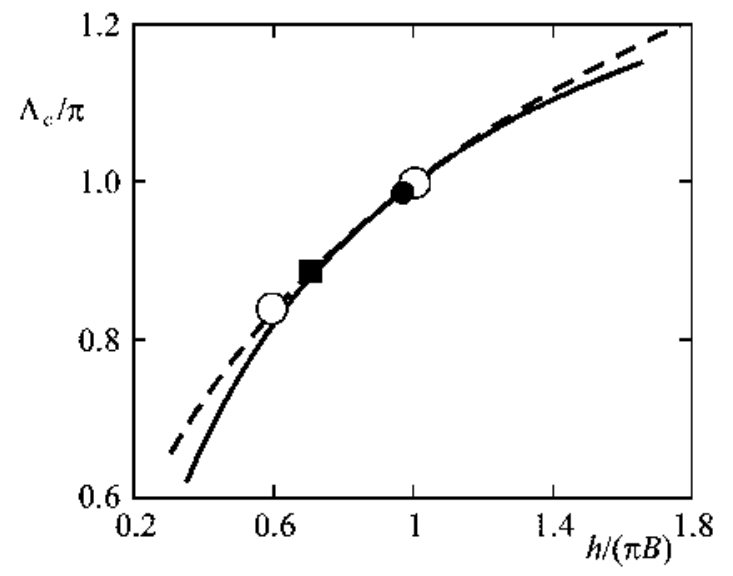

Fig. 5. Variation with the ratio $h /(\pi B)$ between the parameter $h$ that measures the disks inequality and the Bond number $B$, of the reduced slenderness $\lambda_{c} / \pi$ at the point where a local minimum volume is reached. Solid line correspond to the results provided by cxpression (12) whereas the dashed one represents exact theoretical results $[21,22]$. Black symbols indicate experimental results obtained by Bezdenejnykh and Meseguer [20] (circles) and by Perales, Meseguer and Martínez $|21|$ (square), whercas open symbols indicate experimental results here obtained.

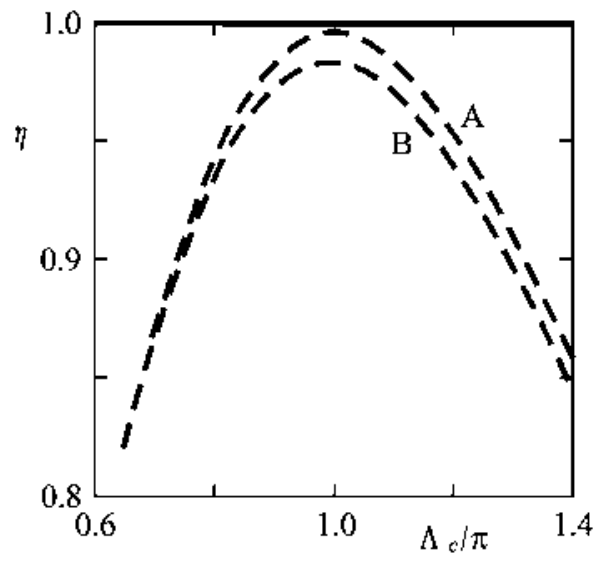

l.ig. 6. Variation of the ratio $\eta=2 \Lambda_{c} /\left|\pi\left(V_{c}+1\right)\right|$ with the reduced slendemess $\Lambda_{c} / \pi$ at the point where a local minimum volume $V_{t}$ : is reached. Solid line $\eta=1$ is the solution of the asymptotic analysis (cxpression (13)) whereas dashed lines represent exact theoretical results $[21,22]$. The letters on the curves identify the liquid bridge configuration according to the following key: (A) $B=0.048, h=0.09$; (B) $B=0.063, h=0.20$.

Table 1

Propertics of the different experimental configurations used to determine the peak of local minimum volume. $\Lambda$ and $B$ indicate the liquid bridge configurations tested in this paper whereas [20] and [21] identify the publications where the other configurations were used

\begin{tabular}{cccccc}
\hline Configuration & $h$ & $B$ & $h /(\pi B)$ & $\Lambda_{c} / \pi$ & Comments \\
\hline A & 0.0909 & 0.048 & 0.60 & 0.84 & Millimetric liquid bridges \\
B & 0.2000 & 0.063 & 1.01 & 1.00 & Millimeiric liquid bridges \\
{$[20 \mid$} & 0.25 & 0.080 & 0.99 & 0.99 & Millimetric liquid bridges \\
{$[21]$} & 0.10 & 0.046 & 0.69 & 0.88 & Plateau tank (neutral buoyancy) technique \\
\hline
\end{tabular}

It is clear that at the peak $\left(\Lambda_{C}, V_{C}\right)$ the root of $\mathrm{d} \lambda / \mathrm{d} \varepsilon=0$ is $\varepsilon=0$, and then, from expression (9) the following relationships between $\Lambda_{C}, V_{c}, B$ and $h$ are obtained:

$$
\begin{aligned}
& \frac{\Lambda_{c}}{\pi}=\frac{3 h /(\pi B)}{1+2 h /(\pi B)}, \\
& \frac{\Lambda_{c}}{\pi}=\frac{1}{2}\left(V_{c}+1\right) .
\end{aligned}
$$

Therefore, within the scope of this asymptotic analysis, the peak $\left(\Lambda_{c}, V_{c}\right)$ is only a function of the ratio $h / B$, no matter what the values of $h$ and $B$ are. Expressions (12) and (13) are compared with exact numerical results [21,22] in Figs. 5 and 6 , respectively. In Fig. 5 the experimental results here obtained are represented, along with other experimental results already published which were obtained working with millimetric liquid bridges [20] or using the neutral buoyancy (Plateau tank) technique [21]. The different experimental conditions are summarized in Table 1.

\section{Experimental analysis}

The main aim of the experiments is to determine the relative volume of the two resulting drops after the breakage of the liquid bridge. This ratio is determined by integrating the liquid bridge outer shape from either disk until the neck position for a video frame taken just before breakage. 


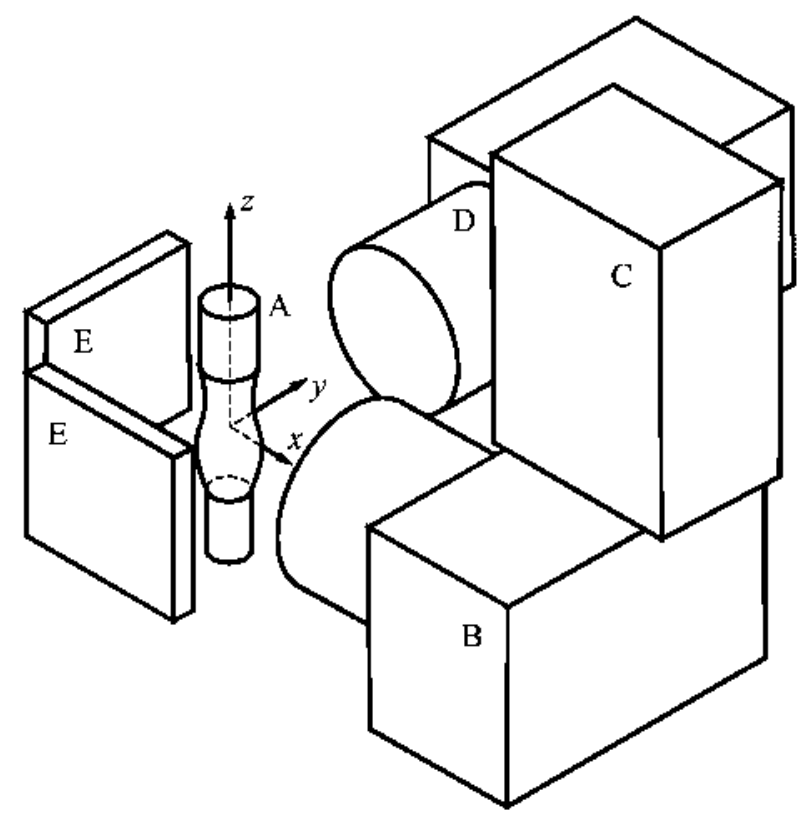

Fig. 7. Experimental set-up: liquid bridge cell (A), microscope (B), high speed CCD camera (C), normal rate CCD camera (D), and illumination system (E).

The experimental set-up used to perform the experiments, as sketehed, not to scalc, in Fig. 7, consists of the following main clements: liquid bridge cell (A), microscope (B), high specd video camera (C), normal rate CCD camcra (D), illumination system (E), image recording system (not shown in the figurc) and associated software.

The liquid bridge cell consists of a three-axes table, the displacement along each one of the different axes being controlled through micrometric screws. The bottom disk is mounted on the horizontal platform of the three-axes table, and it can be displaced both along the $x$-axis and the $y$-axis. The upper disk is mounted on the vertical guide of the table, so that its displacement can only be along the z-axis. Liquid bridge supports (disks) are made of calibrated stainless-steel tube, their surfaces in contact with the working liquid being carefully polished to provide very sharp edges. One of these tubes is used for feeding and removing liquid, whereas the hole on the second one is sealed to avoid undesirable and unconirolled changes of the liquid bridge volume during experimentation. The disks diameters used in experiments have been $1.0,1.2 \mathrm{and} 1.5 \mathrm{~mm}$.

There is a CCD camera (SONY CCD-IRIS) with its optical axis aligned with the $y$-axis of the liquid bridge cell (D). This camera is used to visualize the liquid bridge during the liquid column formation phase, providing enlarged images of the liquid bridge in a user-friendly format, through the corTesponding TV monitor. The camera has its own illumination system, consisting of a background screen illuminated from the rear by a cool light source. The light source is far from the liquid bridge cell, the light being conducted through optic-fiber cables.

There is a second CCD camera (C) whose optical axis is aligned with the $x$-axis. This second CCD camera is a highspeed camera (HISIS 2002) which can record up to 2400 frames per second. All the breaking scquences have been recorded at 1220 images per second, which is the maximum recording speed for which full resolution images can be taken (the resolution decreases as the recording speed increases above this threshold). The high specd CCD camera is mounted on a microscope (WILD M8). The illumination system for the high specd camera consists of a background LED stroboscope (9 LEDs arranged in a matrix of 3 by 3 ) driven by HISIS 2002 system. The stroboscope illuminates the liquid column from the rear (as seen from the high speed camera), so that the liquid-air interface becomes plainly visible (the liquid column, and specially its edges, turns out black since the liquid bridge acts like a small lens).

The experimental proceeds as follows: once the supporting tubes are installed with their longitudinal axes aligned, the upper disk is placed a given distance apart from the lower one. Then a drop of water is injected through the feeding tube and its volume increased until the drop becomes in contact with the opposite disk and a liquid bridge is formed. The amount of liquid supplied to the liquid bridge is always high enough to assure that the volume of the initial drop is far enough from the minimum-volume stability limit corresponding to the selected slenderness. The supports are separated to the desired slenderness, additional volume of liquid being injected, if necessary, to keep the liquid bridge volume within stable boundaries during the whole formation process. 
Once the liquid bridge is formed, the experimental sequence proceeds without any further interaction: due to liquid evaporation, the volume of liquid continuously decreases, and when the stability limit is reached the liquid column breaks. Interface evolution due to evaporation [39] is much slower (some minutes) than the breakage due to capillary instability that suddenly developes (within a single high-speed video frame, $0.001 \mathrm{~s}$ ). All along the breaking sequence, liquid bridge pictures obtained from HISIS 2002 CCD camera, at a rate of 1220 frames per second, are stored in a PC computer. When the operator realizes that the breakage of the liquid column has occurred, the recording process is stopped, and the last part of the breaking sequence is stored for further analysis (about two hundred images prior to breaking time). Afterwards a new value of the slenderness is set, and the whole process is repeated.

The value of the Bond number during the different set of experiments has been calculated by analyzing the interface deformation of liquid bridges between equal disks with dimensionless volumes close to $V=1$. According to this procedure,
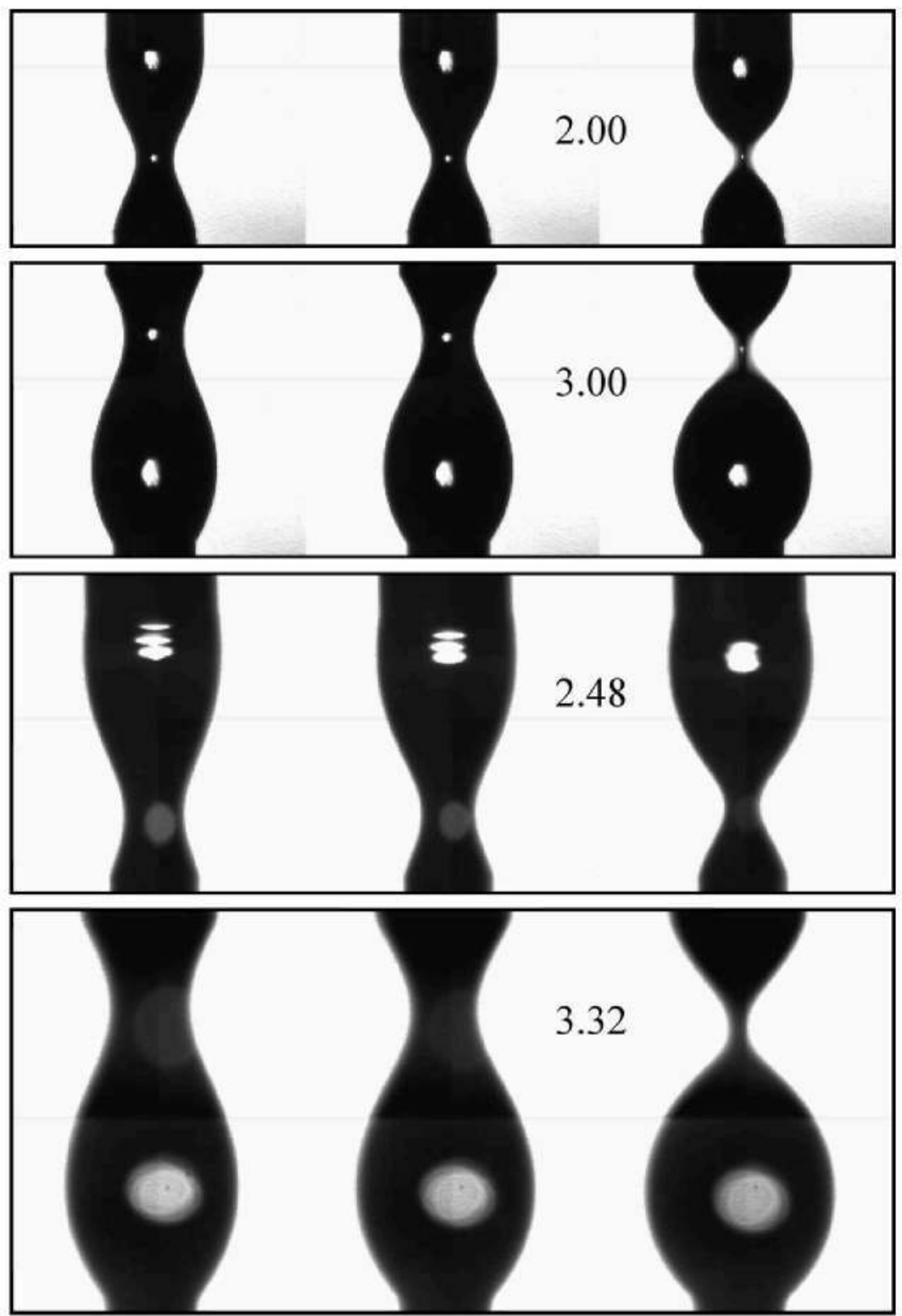

Fig. 8. Consecutive video frames of the liquid bridge just before breaking obtained from high speed camera pictures (the time interval between contours is $0.005 \mathrm{~s}$ ). First and second picture series correspond to the configuration $B=0.048, h=0.09$, whilst third and fourth correspond to $B=0.063, h=0.20$. The numbers indicate the value of the slenderness $A$. 
already uscd in previous expcrimental studies [20,39,41-43], since liquid bridges are formed with an initial volume higher than the cylindrical one $(V>1)$, there are always several images of the liquid bridge with almost cylindrical volume $(V \approx 1)$. These images are selected, stored and digitized. Some video frames are shown in Fig. 8. As il can be observed, the contrast between the liquid bridge and the background is very high and the position of the interface can be easily determined by looking, for every horizontal line in the video frame the position where a given threshold in pixel intensity following a similar procedure to the one described in [42|. The volume of the liquid bridge can be then computed from the digitized outer shapes. From those liquid column contours which volume is within the range $V=1 \pm 0.005$ the value of the l3ond number is computed. [ lence the value of the surface tension is calculated by fitting a linear-order theoretical expression for the interface shape to that experimentally measured. The same procedure is repeated for different values of the slenderness, the result obtained being $B=0.04\left(\sigma=0.062 \mathrm{~N} \cdot \mathrm{m}^{-1}\right)$ for liquid bridges held between disks having $0.50 \mathrm{~mm}$ in radius. This value is very close to the reference value ( $B=0.034$ ) which is obtained using the nominal values of the physical magnitudes and entering the Bond number definition $\left(g=9.81 \mathrm{~m} \cdot \mathrm{s}^{-2}, \rho=10^{3} \mathrm{~kg} \cdot \mathrm{m}^{-3}\right.$, and $\sigma=0.073 \mathrm{~N} \cdot \mathrm{m}^{-1}$ ). The difference in surface tension is thought to be due to the decrease in the value of the surface tension, due to interface contamination. No particular care was taken in avoiding liquid contamination as the Bond number was determined as part of the analysis. Bond number was determined analyzing ten different sequences, the differences being negligible.

Two liquid bridge configurations have been experimentally tested. In both configurations the botlom disk is $1 \mathrm{~mm}$ in diameter whereas the top one is either $1.2 \mathrm{~mm}$ (configuration $\mathrm{A}$ ) or $1.5 \mathrm{~mm}$ in diameter (configuration $\mathrm{B}$ ). Therefore, the values of the Bond number and disks incquality arc $B=0.048, h=0.09$ for configuration $\mathrm{A}$, and $B=0.063, h=0.20$ for configuration $B$. The experimental stability limits measured with these two experimental configurations (minimum volume, $v$, versus slenderness, $A$ ) are represented in Figs. 9 and 10. In these plots black symbols indicate that when the liquid bridge breaks into two drops, the larger drop is formed at the top, larger disk, whereas open symbols indicate that the larger drop is formed at the bottom, smaller disk. Solid lines in both plots represent exact theoretical results [21,22]. As it can be observed, in cach plot experimental results are scattered around the theoretical curve corresponding to the nominal value of the Bond number, the scattering increasing as the slenderness increases. Note that for values of the slenderness smaller than $A_{c}$ the larger drop is at the top disk, as already said, which means that for $A<A_{c}$ the unequal disks effect is the dominant one, as predicted by

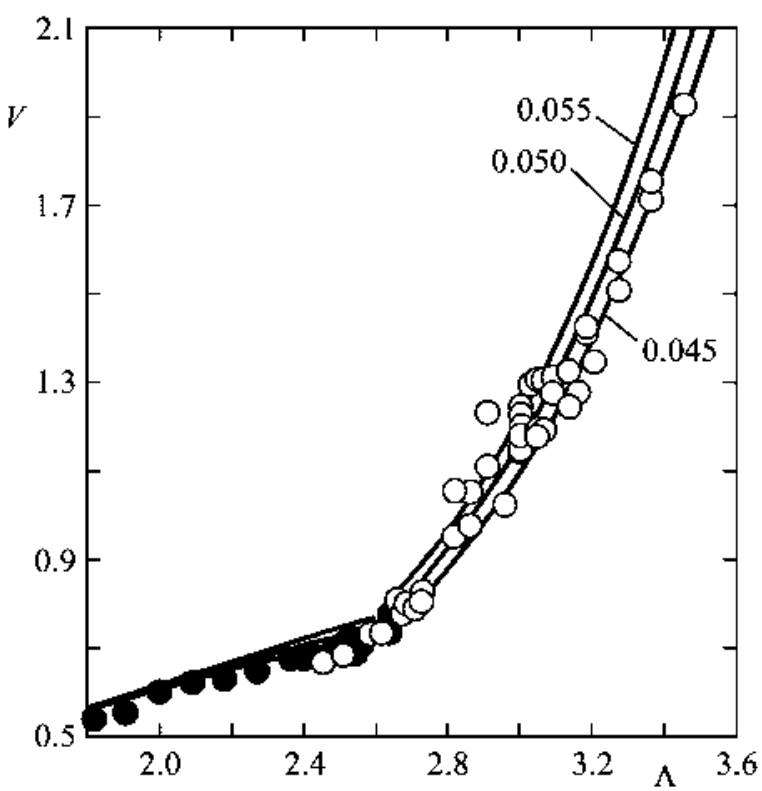

Fig. 9. Fxperimental results: variation with the slenderness $A$ of the minimum volume $V$ of liquid bridges between unequal disks and subject to an axial Bond number (configuration $A: B=0.048$, $h=0.09$ ). Black symbols indicate that when the liquid bridge breaks the larger drop is formed at the top disk, whereas open symbols indicate that the larger drop is at the bottom disk. Solid lines represent exact theoretical results $[21,22]$ for the same disks configuration. The numbers on the curves indicate the value of the Bond number $B$ used to obtain theoretical results.

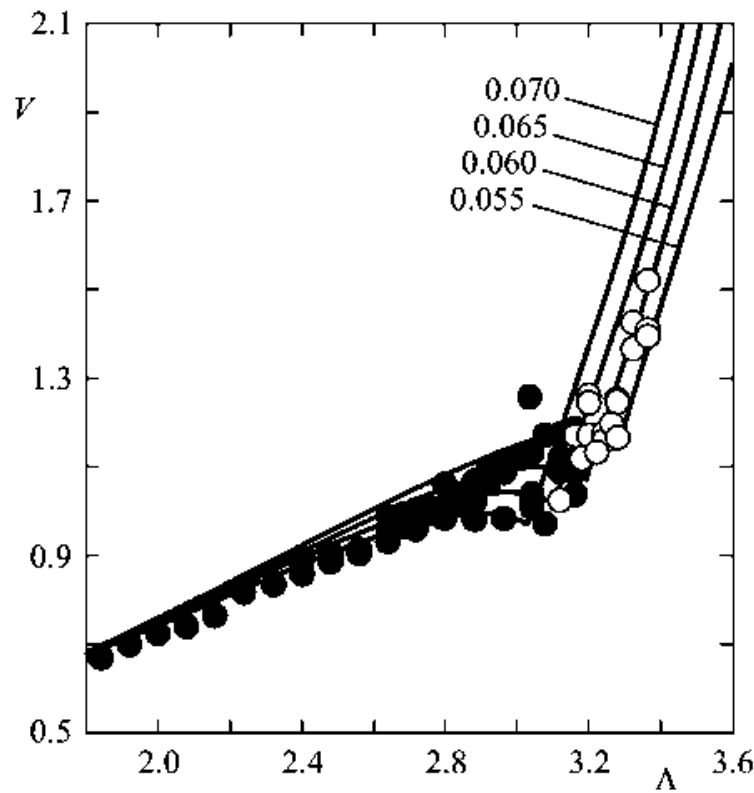

Fig. 10. Experimental results: variation with the slenderness $A$ of the minimum volume $V$ of liquid bridges between uncqual disks and subject to an axial Bond number (configuration $B: B=0.063$, $h=0.20$ ). Black symbols indicate that when the liquid bridge breaks the larger drop is formed at the top disk, whereas open symbols indicate that the larger drop is at the bottom disk. Solid lines represent exact theoretical results $[21,22]$ for the same disks configuration. The numbers on the curves indicate the value of the Bond number $B$ used to obtain theoretical results. 


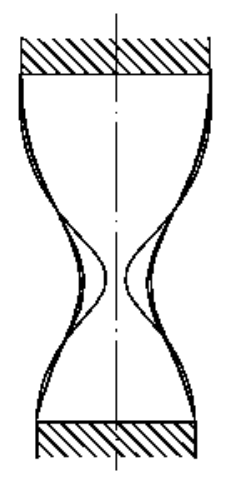

2.00
A

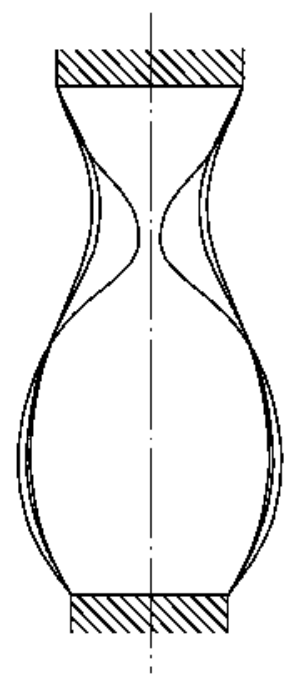

3.00

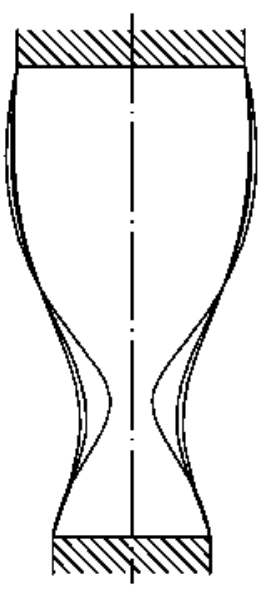

2.48

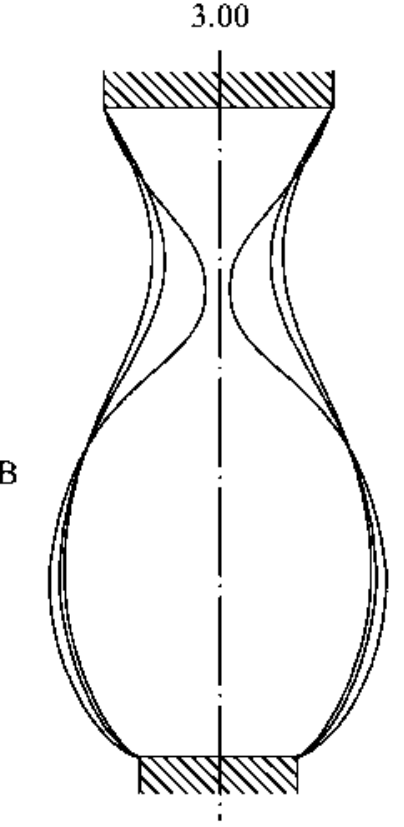

3.32

Fig. 11. Interlace contours of the liquid bridge just before breaking obtained from high speed camera pictures shown in Fig. 8 (the lime interval between contours is $0.005 \mathrm{~s}$ ). Letters on the sketches identify the fluid configuration according to the following kcy: $(\Lambda) B=0.048, h=0.09$; (B) $B=0.063, h=0.20$. The numbers indicate the value of the slenderness $A$.

asymptotic analysis, whereas the contrary happens when $A>\Lambda_{c}$. In this case the dominant effect is the Bond number and the larger drop is formed at the bottom disk.

Liquid bridge contours for configurations very close to the rupture of the liquid column are shown in Fig. 11. In each sketch the contour corresponding to the last image taken by the high speed camera prior to the breakage has been plotted. Taking into account the recording speed, $1 / 1200 \mathrm{~s}$ after there will be two drops of liquid instead of a liquid bridge. Time interval between different contours shown in each sketch is $0.005 \mathrm{~s}$, thence there are six more frames between one contour and the next one represented. These plots clarify the breaking dynamics of the liquid bridge as the fluid configuration moves along the minimum volume stability limit; as $A$ grows the neek of the liquid column displaces from the lower half of the bridge to the upper one, so that the relative volume of the drops varies aceordingly.

The relative volumes have been calculated integrating the liquid bridge contours from the liquid bridge neck to the upper disk, using the last liquid bridge obtained before the breakage. $\Lambda$ s explained before, between the frame analyzed and the broken 


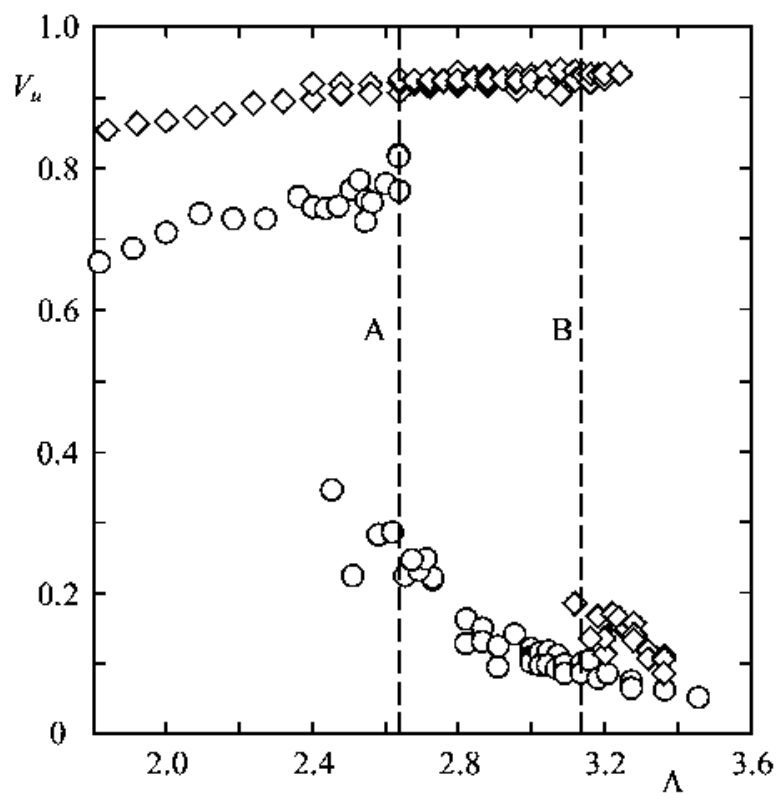

Fig. 12. Experimental results: variation with the slenderness $A$ of the relative volume of the drop formed at the top disk, $V_{u}$. The results correspond to liquid bridges held between unequal disks and subject to an axial Bond number. Circles represent experimental results corresponding to configuration $\Lambda\left(B=0.048, h=0.09, A_{c}=2.64\right)$, whercas rhombi correspond to configuration $\mathrm{B}(B=0.063, h=0.20$, $\left.\Lambda_{c}=3.14\right)$.

into two drops liquid bridge there is less than $0.001 \mathrm{~s}$ and, as velocities in the neck are not too large, the change in the volume of the resulting drops compared with that calculated with this procedure is negligible. The variation with the slenderness $\Lambda$ of the relative volume $V_{u}$ of the drop anchored to the upper disk has been represented in Fig. 12 (similar experimental results were obtained by Bezdenejinykh and Meseguer |20|). Note that the relative volume changes drastically for a critical value $A_{C}$. Obviously, since the value of $A_{c}$ depend on the value of the Bond number, and Bond number depends in turn on the value of the surface tension, leaving apart other effects that could affect the liquid bridge breakage (i.e., uncontrolled external perturbations) there is some scaltering on the relative volume $V_{u}$ very close to the nominal value of $A_{c}$, and the larger drop is formed either at the upper or at the bottom disk depending of the relative importance of the two effects under consideration (unequal disks and Bond number). The theoretical prediction given by expression (12) has also been represented in Fig. 12.

In conclusion, the dynamic bchavior of liquid bridges at minimum volume stability limit has becn analyzed by using an asymptotic approach that, in spite of its simplicity, accurately predicts the response of liquid bridges when the stability limit of minimum volume is reached. Asymptotic results have been compared with exact theoretical results $[21,22]$, the agreement being satisfactory. In addition, to check theoretical predictions, an experimental set-up has been developed and several series of experiments on the breaking of liquid bridges at minimum volume stability limit have been performed, the agreement between experimental results and theoretical ones, either exact or asymptotic, being also satisfactory. Particularly it must be pointed out the excellent agrecment between theoretical and experimental results concerning the propertics of the position of the local minimum in volume in the stability limit shown in Fig. 5 .

\section{Acknowledgements}

This work has been supported by the Spanish Ministerio de Ciencia y Tecnologia (MCyT) under grant ESP2001-4515-PE.

\section{References}

|1] J.M. Haynes, Stability of a fluid cylinder, J. Colloid Interface Sei. 32 (1970) 652-654.

[2] M.A. Lile, R.D. Gillette, D.C. Dyson, Stability of interface of revolution with constant surface tension. The case of catenoid, Chem. Eng. J. 1 (1970) 97-109.

[3] R.D. Gillette, D.C. Dyson, Stability of fluid inteffaces of revolution between equal solid circular plates, Chem. Eng. J. 2 (1971) $44-54$. 
[4] I. Martínez, Floating zones. Equilibrium shapes and stability criteria, in: M.J. Rycroft. A.C. Stickland (Eds.), COSPAR Space Research XVIII, Pergamon Press, Oxford, 1978, pp. 519-522.

[5] A. Sanz, I. Martinez, Minimun volume for a liquid bridge between equal disks, J. Colloid Interface Sci. 93 (1983) $235-240$.

[6] M.J. Russo, P.H. Steen. Instability of rotund capillary bridges to general disturbances: experiment and theory, J. Colloid Interface Sci. 113 (1986) 154-163.

[7] E.A. Boucher, T.G.J. Jones, Equilibrium and stability characteristics of zero-gravity fluid bridges constrained between equal solid rods, J. Colloid Interface Sci. 126 (1988) 469-481.

[8] B.J. Lowry, P.H. Steen, Capillary surfaces: stability from families of equilibrium with application to liquid bridge, Proc. Roy. Soc. London Ser. A 449 (1995) 411-439.

[9] L.A. Slobozhanin, J.I.D. Alexander, A.H. Resnick, Bifurcation of the equilibrium states for a weightless liquid bridge. Phys. Fluids 9 (1997) 1893-1905.

[10] E.A. Boucher. M.J.B. Evans, Properties of fluid bridges between solids in a gravitational field, J. Colloid Interface Sei. 75 (1980) $409-418$.

[11] J. Meseguer, A. Sanz Numerical and experinental study of the dyoanics of axisymmetric liquid bridges, J. Fluid Mech. 153 (1985) 83-101.

[12] N.A. Bezdenejnykh. J. Meseguer, J.M. Perales, Experimental analysis of stability limits of capillary liquid bridges, Phys. Fluids A 4 (1992) $677-680$.

[13] L.A. Slobozhanin, J.M. Perales, Stability of liquid bridges between equal disks in an axial gravity field, Phys. Flujds A 5 (1993) $1305-$ 1314.

[14] I. Martínez, J.M. Perales, Liquid bridge stability data, J. Cryst. Growth 78 (1986) 369-378.

[15] L.A. Slobozhanin, M. Gómez. J.M. Perales, Stability of liquid bridges between unequal disks under zero-gravity conditions, Microgravity Sci. Tec. 8 (1995) 23-34.

[16] J. Meseguer, Stability of slender, axisymmetric liquid bridges between uneiulal disks, J. Cryst. Growth 67 (1984) 141-143.

[17] J. Meseguer, The dynamies of axisymmetrie slender liquid bridges between uneejual disks, J. Cryst. Growth 73 (1985) $599-608$.

[18] J. Meseguer, L. Mayo, J.C. Llorente, A. Fernandez, Experiments with liquid bridges in simulated microgravity, J. Cryst. Growth 73 (1985) $609-621$.

[19] J. Meseguer, A. Sanz, J.M. Perales, Axisymmetric long licjuid bridges stability and resonances, Appl. Microgravity Tec. 2 (1990) 186-192.

[20] N.A. Bezdenejnykh, J. Meseguer, Stability linits of nininum volume and breaking of axisymmetric liquid bridges between uneequal disks. Microgravity Sei. Tec. 4 (1991) 235-239.

[21] J.M. Perales, J. Meseguer, I. Martinez, Mininum volume stability linits for axisymmetric liquid bridges subject to steady axial acceleration, J. Cryst. Growth 110 (1991) 855-861.

[22] L.A. Slobozhanin. J.I.D. Alexander, Combined effect of disc inequality and axial gravity on axisymmetric licjuid bridge stability, Phys. Fluids 10 (1998) 2473-2488.

[23] J. Meseguer, J.M. Perales, I. Martinez, N.A. Bezdenejnykh. A. Sanz, Hydrostatic instabilities in floating zone crystal growth process, Currents Topics Cryst. Growth Res. 5 (1999) $27-42$.

[24] J. Meseguer, J.M. Perales, J.I.D. Alexander, A perturbation analysis of the stability of long liquid bridges between almost circular supporting disks, Phys. Fluids 13 (2001) 2724-2727.

[25] B.L. Lowry, P.H. Steen, Stability of slender liquid bridges subjected to axial flows, J. Fluid Mech. 330 (1997) 189-213.

[26] S. Atreya, P.H. Steen, Stability analyses of long liquid bridges in the presence of gravity and flow, Proc. Roy, Soc. London Ser. A 458 (2002) 2645-2669.

[27] M.J. Mart-Lyon. D.B. Thiessen. P.L. Marston, Stabilization of a cylindrical capillary bridge far beyond the Rayleigh-Plateau linit using acoustic radiation pressure and active feedback, J. Fluid Mech. 351 (1997) 345-358.

[28] M.J. Mart-Lyon, D.B. Thiessen, P.L. Marston, Passive stabilitation of capillary bridges in air with acoustic radiation pressure, Phys. Rev. Lett. 86 (2001) 2293-2296.

[29] H. Gonzalez. F.J.M. McCluskey, A. Castellanos. A. Bamero, Stabilization of dielectric liquid bridges by electric fields in the absence of gravity, J. Fluid Mech. 206 (1989) 545-562.

[30] A. Ramos, A. Castellanos, Bifurcation diagrams of axisymmetric liquid bridges of arbitrary volume in electric and gravitational axial fields, J. Fluid Mech. 249 (1993) 207-225.

[31] A. Ramos, H. González, A. Castellanos, Bifurcation diagrams of axisymmetric liquid bridges subjected to axial electric fields, Pliys. Fluids 6 (1994) 3580-3590.

[32] A. Ramos, F.J. Garcia, J.M. Valverde, On the breakup of slender licuid bridges: experiments and a 1-D numerical analysis, Eur. J. Mech. B Fluids 18 (1999) 649-658.

[33] D.B. Thiessen, M.J. Marr-Lyon, P.L. Marston, Active electrostatic stabilization of liquid bridges in low gravity, J. Fluid Mech. 457 (2002) 285-294.

[34] M.P. Mahajan, S. Zhang, M. Tsinge, P.L. Taylor, C. Rosenblatt. Stability of magnetically levitated liquid bridges of arbitrary volume subjected to axial and lateral gravity. J. Colloid Interface Sci. 123 (1999) 592-595.

[35] M.P. Mahajan, M. Tsinge, P.L. Taylor, C. Rosenblatt. Paramagnetic liguid bridge in a gravity-compensating magnetic field, Phys. Fluids 10 (1998) 2208-2211.

[36] J. Meseguer. The breaking of axisymmetric slender liquid bridges, J. Fluid Mech. 130 (1983) 123-151.

[37] W.A. Sirignano, C. Meluring, Review of theory of distortion and disintegration of liquid streans, Prog. Energ. Conbust. 26 (2000) 609 655.

[38] X. Zhang, R.S. Padgett, O.A. Basaran, Nonlinear deformation and breakup of stretching liquid bridges, J. Fluid Mech. 329 (1996) $207-245$. 
[39] J.L. Espino, J. Meseguer, A. Laverón-Simavilla, An experimental study of the breakage of liquid bridges at stability linit of minimum volume, Phys. Fluids 14 (2002) 3710-3713.

[40] N.S. Chow, J.K. Hale. Methods of Bifurcation Theory, Springer-Verlag, Berlin, 1982.

[41] J. Meseguer. N.A. Bezdenejuykh. J.M. Perales, P. Rodriguez de Francisco. Theoretical and experinental analysis of stability limits of non-axisymmetric liquid bridges under microgravity conditions, Microgravity Sci. Tec. 8 (1995) $2-9$.

[42] J.M. Perales, J. Meseguer. Theoretical and experimental study of the vibration of axisymmetric viscous liquid bridges, Phys. Fluids A 4 (1992) 1110-1130.

[43] J. Meseguer, N.A. Bezdenejnykh. P. Rodriguez de Francisco, On the use of liquid bridges as accelerometers, Microgravity Sci. Tec. 9 (1996) 62-69. 